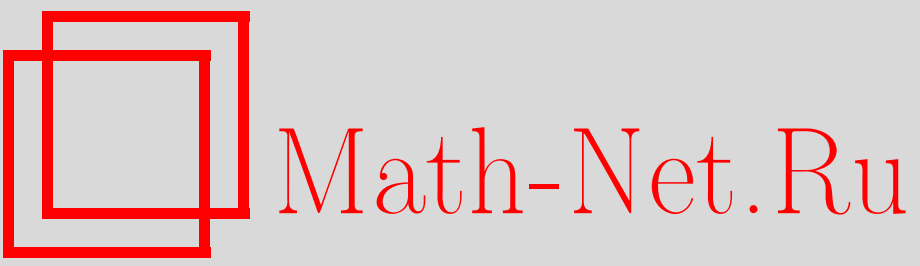

Л. М. Сладь, К теории бесконечнокомпонентных полей с двойной симметрией. Свободные поля, ТМФ, 2001, том 129, номер 1, 68-86

DOI: https://doi.org/10.4213/tmf520

Использование Общероссийского математического портала Math-Net.Ru подразумевает, что вы прочитали и согласны с пользовательским соглашением

http://www.mathnet.ru/rus/agreement

Параметры загрузки:

IP: 3.80 .253 .173

26 апреля 2023 г., 18:04:53 
ТЕОРЕТИЧЕСКАЯ

И МАТЕМАТИЧЕСКАЯ

ФИЗИКА

Том 129, № 1

октябрь, 2001

(C) 2001 г. $\quad$ Л.М. Сладь ${ }^{*}$

\section{К ТЕОРИИ БЕСКОНЕЧНОКОМПОНЕНТНЫХ ПОЛЕЙ С ДВОЙНОЙ СИММЕТРИЕЙ. СВОБОДНЫЕ ПОЛЯ}

Сделан первый шаг на пути исследования возможности описания адронов с помощью монолокальных полей, которые преобразуются по представлениям собственной группы Лоренца, разложимым в бесконечную прямую сумму конечномерных неприводимых представлений. В качестве эффективного механизма отбора представлений названного класса и устранения бесконечного произвола в константах лагранжианов свободных полей, допускаемого релятивистской инвариантностью, служит принимаемое требование инвариантности лагранжианов также относительно преобразований вторичной симметрии, порождаемой полярным или аксиальным 4-векторным представлением ортохронной группы Лоренца.

\section{1. ВВЕДЕНИЕ}

Первая попытка релятивистски-инвариантного описания частиц с внутренними степенями свободы, предпринятая Гинзбургом и Таммом [1], основывалась на билокальных уравнениях. Оказалось, что каждое из рассматривавшихся уравнений дает плохой спектр масс, а именно было показано, что с ростом некоторого квантового числа, характеризующего состояния, массы стремятся к нулю. Чтобы выяснить степень общности этого результата, Гельфанд и Яглом [2] дали полное описание всех линейных релятивистски-инвариантных уравнений вида

$$
\left(\Gamma^{\mu} \partial_{\mu}+i R\right) \Psi(x)=0
$$

где $\Gamma^{\mu}$ и $R$-матричные операторы, и соответствующих им лагранжианов. Ограничившись уравнениями вида (1), в которых поля $\Psi(x)$ преобразуются по представлениям собственной группы Лоренца, разложимым в конечную прямую сумму бесконечномерных неприводимых представлений (в дальнейшем будем говорить, что такие поля принадлежат классу FSIIR), а оператор $R$ невырожден, Гельфанд и Яглом пришли к заключению, что при неограниченном росте спина массы стремятся к нулю. Позднее было показано [3], что это заключение в ряде случаев неверно: сушествуют такие представления собственной группы Лоренца класса FSIIR и такие ограничения на произвольные

${ }^{*}$ Научно-исследовательский институт ядерной физики им. Д.В. Скобельцына, Московский государственный университет, Москва, Россия. E-mail: slad@theory.sinp.msu.ru 
константы уравнения (1), при которых одни ветви спектра масс с ростом спина стремятся к нулю, а другие - к бесконечности. Показано также, что в опущенном в работе [2] случае, когда матричный оператор $R$ является вырожденным, сушествуют такие уравнения типа (1) с полями класса FSIIR, для которых спектр ненулевых масс стремится к бесконечности с ростом спина, но при этом для всех спинов начиная с некоторого минимального $s_{0}$ существуют безмассовые состояния. Отмеченное устранение неполноты заключения Гельфанда и Яглома не меняет его первоначальную физическую сущность: получаемый в рамках лагранжева подхода спектр масс любого из полей класса FSIIR обладает такими особенностями, которые абсолютно неприемлемы в физике частиц.

Достаточно общим свойством полей класса FSIIR является их локальная некоммутативность: $[\Psi(x), \Psi(y)]_{ \pm} \neq 0$, если $(x-y)^{2}<0$, доказанная в работе [4] в предположении полноты системы состояний полей и отсутствия в спектре масс бесконечного вырождения уровней. По мнению авторов монографиии [5] эта трудность, по-видимому, требует ослабления постулата строгой локальности.

Для бесконечнокомпонентных полей, описываемых уравнением (1), может иметь место связь спина и статистики или нарушение такой связи в зависимости от того, на какие неприводимые представления раскладывается представление собственной группы Лоренца (не обязательно класса FSIIR), по которому преобразуется поле [6].

Некоторые из выявленных “болезней” полей класса FSIIR - сушествование пространственноподобных решений уравнений Гельфанда-Яглома [7], отсутствие $C P T$-инвариантности [8] - не являются общими для них (контрпримеры можно найти соответственно в работах [9], [10]).

Итог всех прежних исследований бесконечнокомпонентных полей можно отразить следующими суждениями.

Во-первых, бесперспективность такого описания частиц установлена только для одного класса бесконечнокомпонентных полей - класса FSIIR. До сих пор не были исследованы поля, которые преобразуются по представлениям собственной группы Лоренца, разложимым в бесконечную прямую сумму конечномерных или бесконечномерных неприводимых представлений (в дальнейшем будем говорить, что такие поля принадлежат классу ISFIR или ISIIR, соответственно). Причиной тому является бесконечное количество произвольных констант в релятивистски-инвариантных лагранжианах полей таких классов.

Во-вторых, убедившись, что обшие свойства конечнокомпонентных полей и полей класса FSIIR во многих отношениях не совпадают, мы вправе ожидать, что и общие свойства полей классов FSIIR и ISFIR будут различными.

Эти суждения служат нам почвой для обрашения к исходному интуитивному предположению о том, что существуют такие бесконечнокомпонентные поля, с помощью которых можно получить эффективное описание всех состояний частиц с внутренними степенями свободы (адронов).

В отношении теории полей класса ISFIR, с которыми мы будем иметь дело в настояшей работе, сразу можно заключить следующее. Во-первых, она является CPT-инвариантной (для доказательства этого в духе Паули [11] несушественно, какой являет- 
ся сумма конечномерных неприводимых представлений собственной группы Лоренца конечной или бесконечной). Во-вторых, (анти)коммутатор любых двух компонент полей класса ISFIR выражается в виде конечной суммы производных от функции Паули-Йордана $D(x-y)$, но на множестве всех таких (анти)коммутаторов максимальная степень производных не ограничена.

Чтобы пояснить принципиальное отличие теории конечномерных полей от теории полей класса ISFIR, воспользуемся простой аналогией, достаточно хорошо передающей сушность дела. Если физическим величинам, в первую очередь сечениям, в первой теории можно поставить в соответствие конечные полиномы от некоторой переменной $x$, то во второй - бесконечные сходящиеся ряды. Если коэффициенты в этих рядах знакопеременны, то при $x \rightarrow+\infty$ величины, отвечающие таким рядам, могут иметь, в частности, быстрое стремление к нулю. Роль переменной $x$ в двух указанных теориях играют матричные элементы конечных преобразований неприводимых представлений собственной группы Лоренца, которые даются конечными рядами Лорана от лоренцова фактора $\left(1-v^{2} / c^{2}\right)^{-1 / 2}$. В той теории, к которой мы придем в результате осуществляемого построения, мы действительно будем иметь подобный знакопеременный ряд, в частности, для дифференциального сечения рассеяния заряженного лептона на протоне, если поставим в соответствие протону низшее энергетическое состояние со спином $1 / 2$ одного из наших бесконечнокомпонентных полей (подробное описание этого будет дано в последующих работах).

Отметим еще, что одной из самых серьезных трудностей для теорий частиц с высшими спинами в рамках конечномерных представлений собственной группы Лоренца является проблема перенормируемости теории. Знания об этой трудности никак не приближают нас к ответу на вопрос о перенормируемости какой-то конкретной теории полей класса ISFIR, решение которого несомненно будет иметь крайне высокую степень сложности.

Две наши статьи - настоящая и готовящаяся к изданию - посвящаются детальному описанию подкласса теорий полей класса ISFIR, выделяемому на основании требования, чтобы лагранжианы свободных и взаимодействующих полей обладали двойной симметрией, обшее определение которой дано в работе [12], - релятивистской инвариантностью ${ }^{1)}$ (первичной симметрией) и инвариантностью относительно преобразований глобальной вторичной симметрии, порождаемой полярным или аксиальным 4-векторным представлением группы $L^{\uparrow}$. Эти преобразования можно записать в следующем виде:

$$
\Psi(x) \rightarrow \Psi^{\prime}(x)=e^{-i D^{\mu} \theta_{\mu}} \Psi(x),
$$

где параметры $\theta_{\mu}$ являются компонентами полярного или аксиального 4 -вектора ортохронной группы Лоренца, а $D^{\mu}$ - матричные операторы.

\footnotetext{
1) Релятивистская инвариантность в современных терминологии и обозначениях [5] представляет собой инвариантность относительно ортохронной группы Лоренца $L^{\uparrow}$, порождаемой собственной группой Лоренца $L_{+}^{\uparrow}$ и пространственным отражением $P$. В статье [2] и монографоии [13] группу $L^{\uparrow}$ называют полной группой Лоренца.
} 
Если элементу $g$ группы $L^{\uparrow}$ отвечают преобразования поля $\Psi(x)$ и полярного или аксиального 4-вектора $\theta_{\mu}$, имеющие вид

$$
\begin{gathered}
\Psi^{\prime}(x)=S(g) \Psi(x), \\
\theta_{\mu}^{\prime}=\left[l_{ \pm}(g)\right]_{\mu}{ }^{\nu} \theta_{\nu},
\end{gathered}
$$

где знаки “+" и “-” отвечают соответственно преобразованиям полярного и аксиального 4-вектора $\theta_{\mu}$, то операторы $D^{\mu}$ должны удовлетворять соотношениям

$$
S^{-1}(g) D^{\nu} S(g)\left[l_{ \pm}(g)\right]_{\nu}^{\mu}=D^{\mu}
$$

Каковы наши физические и математические мотивы обращения к вторичной симметрии вообще и к данной конкретной в частности? Во-первых, помимо первичной симметрии, задаваемой свойствами пространства-времени, материя может обладать своей собственной симметрией, которая связана с первичной симметрией, согласуется с ней и не нарушает ее. Во-вторых, используя современные представления о внутренней структуре адронов, преобразование (2) с полярным 4-вектором $\theta_{\mu}$ можно интерпретировать как добавление к адрону (поле $\Psi(x))$ некоторого когерентного состояния (экспонента) глюонов и кварк-антикварковых пар. Кроме того, мы на равных основаниях будем рассматривать преобразования (2) с аксиальным 4-вектором $\theta_{\mu}$ и к тому же будем ограничиваться глобальными преобразованиями. В-третьих, поскольку все конечномерные неприводимые представления собственной группы Лоренца с цельми спинами можно получить из прямых произведений 4-векторного представления, то ограничения на теорию, накладываемые рассматриваемой вторичной симметрией, уступают по жесткости только ограничениям, которые давала бы вторичная симметрия, порождаемая биспинорным представлением собственной группы Лоренца. Последние ограничения подробно нами рассмотрены, но здесь не приводятся.

Как будет показано в настоящей работе, требование инвариантности лагранжиана свободного поля относительно преобразований вторичной симметрии (2) в дополнение к его релятивистской инвариантности служит эффективным инструментом отбора представлений собственной группы Лоренца и устранения бесконечного произвола в константах лагранжиана. При этом уровень симметрии каждого из получаемых уравнений (1) столь высок, что спектр масс частиц бесконечно вырожден по спину, непрерывен и простирается от некоторого положительного значения $m_{0}$ до $+\infty$, если $R \neq 0$. Чтобы снять это вырождение, мы будем предполагать, что вторичная симметрия спонтанно нарушена, т.е. что одна или несколько лоренцевых скалярных компонент рассматриваемых бесконечнокомпонентных полей имеют ненулевое вакуумное среднее. Аналогом этого в квантовой хромодинамике является предположение о кварк-антикварковом конденсате. Итак, прежде чем приступать к исследованию свойств выделяемого класса свободных полей, необходимо вначале найти структуру лагранжианов взаимодействия таких полей, обладающих требуемой двойной симметрией. Эта задача будет предметом нашей готовяшейся к изданию работы. 


\section{2. НЕОБХОДИМЫЕ СВЕДЕНИЯ О ПРЕДСТАВЛЕНИЯХ ГРУППЫ ЛОРЕНЦА И О РЕЛЯТИВИСТСКИ-ИНВАРИАНТНЫХ ЛАГРАНЖИАНАХ}

Представляется целесообразным дать вначале являющиеся основой для выкладок настоящей работы сведения о неприводимых представлениях группы Лоренца и о релятивистски-инвариантных лагранжианах, почерпнутые из статьи [2] и монографии [13]. Это к тому же устранит возможные неудобства, связанные с давностью издания названных работ, установит однозначность понятий и обозначений, тем более что до сих пор в литературе при описании представлений превалирует замена группы Лоренца на группу $S O(4)=S O(3) \otimes S O(3)$.

Бесконечно малому собственному преобразованию Лоренца контравариантных координат пространства-времени

$$
x^{\mu} \rightarrow x^{\prime \mu}=x^{\mu}+g^{\mu \nu} \epsilon_{\nu \rho} x^{\rho}
$$

где $g^{00}=-g^{11}=-g^{22}=-g^{33}=1, g^{\mu \nu}=0(\mu \neq \nu), \epsilon_{\nu \rho}=-\epsilon_{\rho \nu}$, ставится в соответствие преобразование в пространстве представления

$$
\Psi \rightarrow \Psi^{\prime}=\Psi+\frac{1}{2} \epsilon_{\mu \nu} I^{\mu \nu} \Psi
$$

причем $I^{\mu \nu}=-I^{\nu \mu}$.

Коммутационные соотношения для инфинитезимальных операторов собственной группы Лоренца имеют вид

$$
\left[I^{\mu \nu}, I^{\rho \sigma}\right]=-g^{\mu \rho} I^{\nu \sigma}+g^{\mu \sigma} I^{\nu \rho}+g^{\nu \rho} I^{\mu \sigma}-g^{\nu \sigma} I^{\mu \rho}
$$

Неприводимое представление $\tau$ собственной группы Лоренца определяется парой чисел $\left(l_{0}, l_{1}\right)$, где $2 l_{0}$ - целое, а $l_{1}$ - произвольное комплексное число. Канонические базисные векторы пространства этого представления связываются с подгруппой $S O(3)$ и обозначаются через $\xi_{\tau l m}$, где $l$ - спин, $m$ - его проекция на третью ось, причем $m=-l,-l+1, \ldots, l ; l=\left|l_{0}\right|,\left|l_{0}\right|+1, \ldots$. Представление $\tau=\left(l_{0}, l_{1}\right)$ конечномерно, если $2 l_{1}$ - целое число одинаковой четности с $2 l_{0}$ и $\left|l_{1}\right|>\left|l_{0}\right|$, тогда $l=\left|l_{0}\right|,\left|l_{0}\right|+1, \ldots,\left|l_{1}\right|-1$. Пары $\left(l_{0}, l_{1}\right)$ и $\left(-l_{0},-l_{1}\right)$ определяют одно и то же представление: $\left(l_{0}, l_{1}\right) \sim\left(-l_{0},-l_{1}\right)$. Если ввести операторы

$$
F^{3}=i I^{30}, \quad F^{-}=i I^{10}+I^{20}, \quad F^{+}=i I^{10}-I^{20},
$$

то для неприводимого представления $\tau=\left(l_{0}, l_{1}\right)$ справедливы формулы

$$
\begin{aligned}
F^{3} \xi_{\tau l m}= & B_{\tau l} \sqrt{l^{2}-m^{2}} \xi_{\tau l-1 m}-A_{\tau l} m \xi_{\tau l m}-B_{\tau l+1} \sqrt{(l+1)^{2}-m^{2}} \xi_{\tau l+1 m} \\
F^{-} \xi_{\tau l m}= & -B_{\tau l} \sqrt{(l+m)(l+m-1)} \xi_{\tau l-1 m-1}-A_{\tau l} \sqrt{(l+m)(l-m+1)} \xi_{\tau l m-1}- \\
& -B_{\tau l+1} \sqrt{(l-m+1)(l-m+2)} \xi_{\tau l+1 m-1}, \\
F^{+} \xi_{\tau l m}= & B_{\tau l} \sqrt{(l-m)(l-m-1)} \xi_{\tau l-1 m+1}-A_{\tau l} \sqrt{(l-m)(l+m+1)} \xi_{\tau l m+1}+ \\
& +B_{\tau l+1} \sqrt{(l+m+1)(l+m+2)} \xi_{\tau l+1 m+1},
\end{aligned}
$$


где

$$
A_{\tau l}=\frac{i l_{0} l_{1}}{l(l+1)}, \quad B_{\tau l}=\frac{i}{l} \sqrt{\frac{\left(l^{2}-l_{0}^{2}\right)\left(l^{2}-l_{1}^{2}\right)}{4 l^{2}-1}} .
$$

При переходе от собственной к ортохронной группе Лоренца требуется ввести оператор пространственного отражения $P$, действие которого на векторы канонического базиса дается формулой

$$
P \xi_{\tau l m}=(-1)^{[l]} p_{\dot{\tau} \tau} \xi_{\dot{\tau} l m},
$$

где $\dot{\tau}=\left(-l_{0}, l_{1}\right) \sim\left(l_{0},-l_{1}\right)$, когда $\tau=\left(l_{0}, l_{1}\right)$, причем $p_{\dot{\tau} \tau} p_{\tau \dot{\tau}}=1$.

Обычно, независимо от того, рассматривается ли собственная или ортохронная группа Лоренца, указывается представление собственной группы Лоренца.

Если представление собственной группы Лоренца разложимо в конечную или бесконечную прямую сумму конечномерных неприводимых представлений, то для векторов $\Psi_{1}=\left\{\left(\psi_{1}\right)_{\tau l m}\right\}$ и $\Psi_{2}=\left\{\left(\psi_{2}\right)_{\tau l m}\right\}$ из пространства этого представления эрмитова билинейная форма

$$
\overline{\Psi_{2}} \Psi_{1} \equiv\left(\Psi_{2}, \Psi_{1}\right)=\sum_{\tau, l, m, \tau^{\prime}, l^{\prime}, m^{\prime}}\left(\psi_{2}\right)_{\tau^{\prime} l^{\prime} m^{\prime}}^{*} a_{\tau^{\prime} l^{\prime} m^{\prime}, \tau l m}\left(\psi_{1}\right)_{\tau l m}
$$

будет инвариантной относительно собственной группы Лоренца, если

$$
a_{\tau^{\prime} l^{\prime} m^{\prime}, \tau l m}=(-1)^{[l]} a_{\dot{\tau} \tau} \delta_{\tau^{\prime} \dot{\tau}} \delta_{l l^{\prime}} \delta_{m m^{\prime}}
$$

Эрмитовость билинейной формы означает, что $a_{\dot{\tau} \tau}=a_{\tau \dot{\tau}}^{*}$. Эта форма будет инвариантной относительно пространственного отражения $P$, если $a_{\dot{\tau} \tau}=p_{\dot{\tau} \tau}^{*} a_{\tau \dot{\tau}} p_{\dot{\tau} \tau}$.

Если элементу $g$ ортохронной группы Лоренца соответствуют преобразование ковариантных координат пространства-времени вида

$$
x_{\mu}^{\prime}=\left[l_{+}(g)\right]_{\mu}^{\nu} x_{\nu}
$$

и преобразование поля вида (3), то свободный лагранжиан

$$
\mathcal{L}_{0}=\frac{i}{2}\left[\left(\Psi, \Gamma^{\mu} \partial_{\mu} \Psi\right)-\left(\partial_{\mu} \Psi, \Gamma^{\mu} \Psi\right)\right]-\kappa(\Psi, \Psi)
$$

будет релятивистски-инвариантным, если матричные операторы $\Gamma^{\mu}$ удовлетворяют условиям

$$
S^{-1}(g) \Gamma^{\nu} S(g)\left[l_{+}(g)\right]_{\nu}^{\mu}=\Gamma^{\mu} .
$$

Кроме того, оператор $\Gamma^{0}$ должен подчиняться соотношению

$$
\left(\Gamma^{0} \Psi_{1}, \Psi_{2}\right)=\left(\Psi_{1}, \Gamma^{0} \Psi_{2}\right),
$$

чтобы лагранжиан был вешественным. 
Из (13) следует

$$
\begin{aligned}
{\left[I^{\mu \nu}, \Gamma^{\rho}\right] } & =-g^{\mu \rho} \Gamma^{\nu}+g^{\nu \rho} \Gamma^{\mu}, \\
{\left[P, \Gamma^{0}\right] } & =0,
\end{aligned}
$$

в частности

$$
\left[I^{i 0}, \Gamma^{0}\right]=\Gamma^{i}, \quad i=1,2,3
$$

Пусть

$$
\Gamma^{0} \xi_{\tau l m}=\sum_{\tau^{\prime} l^{\prime} m^{\prime}} c_{\tau^{\prime} l^{\prime} m^{\prime}, \tau l m} \xi_{\tau^{\prime} l^{\prime} m^{\prime}}
$$

Тогда условия (15) дают

$$
c_{\tau^{\prime} l^{\prime} m^{\prime}, \tau l m}=c_{\tau^{\prime} \tau}(l) \delta_{l l^{\prime}} \delta_{m m^{\prime}}
$$

причем величина $c_{\tau^{\prime}}(l) \equiv c\left(l \mid l_{0}^{\prime}, l_{1}^{\prime} ; l_{0}, l_{1}\right)$ может быть отличной от нуля для данного $\tau=\left(l_{0}, l_{1}\right)$, если $\tau^{\prime}=\left(l_{0} \pm 1, l_{1}\right)$ или $\tau^{\prime}=\left(l_{0}, l_{1} \pm 1\right)$. Из условия (15) также находятся соотношения

$$
\begin{aligned}
& c\left(l \mid l_{0}+1, l_{1} ; l_{0}, l_{1}\right)=c\left(l_{0}+1, l_{1} ; l_{0}, l_{1}\right) \sqrt{\left(l+l_{0}+1\right)\left(l-l_{0}\right)}, \\
& c\left(l \mid l_{0}, l_{1} ; l_{0}+1, l_{1}\right)=c\left(l_{0}, l_{1} ; l_{0}+1, l_{1}\right) \sqrt{\left(l+l_{0}+1\right)\left(l-l_{0}\right)}, \\
& c\left(l \mid l_{0}, l_{1}+1 ; l_{0}, l_{1}\right)=c\left(l_{0}, l_{1}+1 ; l_{0}, l_{1}\right) \sqrt{\left(l+l_{1}+1\right)\left(l-l_{1}\right)}, \\
& c\left(l \mid l_{0}, l_{1} ; l_{0}, l_{1}+1\right)=c\left(l_{0}, l_{1} ; l_{0}, l_{1}+1\right) \sqrt{\left(l+l_{1}+1\right)\left(l-l_{1}\right)},
\end{aligned}
$$

где $c\left(l_{0}^{\prime}, l_{1}^{\prime} ; l_{0}, l_{1}\right) \equiv c_{\tau^{\prime} \tau}-$ произвольные величины.

Условие (16) сводится к виду

$$
c_{\tau^{\prime} \dot{\tau}} p_{\dot{\tau} \tau}=p_{\tau^{\prime} \dot{\tau}^{\prime}} c_{\dot{\tau}^{\prime} \tau}
$$

а соотношение (14) дает

$$
c_{\dot{\tau} \tau^{\prime}}^{*}(l) a_{\dot{\tau} \tau}=a_{\tau^{\prime} \dot{\tau}^{\prime}} c_{\dot{\tau}^{\prime} \tau}(l)
$$

Из формул (20)-(23) вытекает, что в релятивистски-инвариантном лагранжиане невозможно зацепление конечномерных и бесконечномерных представлений группы Лоренша, так как $c_{\tau^{\prime} \tau}(l)=c_{\tau \tau^{\prime}}(l)=0$ для пар $\tau$ и $\tau^{\prime}$ такого типа: если $\tau=\left( \pm\left|l_{0}\right|,\left|l_{0}\right|+1\right)$, то $\tau^{\prime}=\left( \pm\left(\left|l_{0}\right|+1\right),\left|l_{0}\right|+1\right)$ или $\tau^{\prime}=\left( \pm\left|l_{0}\right|,\left|l_{0}\right|\right)$.

Зная оператор $\Gamma^{0}$, с помошью соотношения (17) находим операторы $\Gamma^{i}, i=1,2,3$. 


\section{3. УСЛОВИЯ, НАКЛАДЫВАЕМЫЕ НА ТЕОРИЮ СВОБОДНЫХ БЕСКОНЕЧНОКОМПОНЕНТНЫХ ПОЛЕЙ ВТОРИЧНОЙ СИММЕТРИЕЙ}

В настояшей работе находятся все варианты теории свободных полей, которая удовлетворяет следуюшим условиям.

УсловиЕ 1. Представление $S$ собственной группы Лоренца, по которому преобразуется любое из рассматриваемых полей, разложимо в бесконечную прямую сумму конечномерных неприводимых представлений, причем кратность каждого из неприводимых представлений не превьшает единицу. Бозонные поля принадлежат одному из двух типов, определяемых транформационными свойствами относительно пространственного отражения: для любого неприводимого представления $\tau$, принадлежащего $S$, либо $p_{\dot{\tau} \tau}=1$, либо $p_{\dot{\tau} \tau}=-1$.

УсловиЕ 2. Лагранжиан (12) каждого поля релятивистски-инвариантен и нерасшепляем, т.е. его нельзя представить в виде суммы двух лагранжианов, не содержаших никаких одинаковых компонент поля.

УСловиЕ 3. Лагранжиан (12) каждого поля инвариантен относительно нетривиальных $\left(D^{\mu} \neq 0\right)$ глобальных преобразований вторичной симметрии $(2)$, порождаемых или полярным, или аксиальным 4-векторным представлением ортохронной группы Лоренца.

УСловиЕ 4. Матричный оператор $\Gamma^{0}$ из лагранжиана (12) является эрмитовым: $c_{\tau^{\prime} \tau}^{*}=c_{\tau \tau^{\prime}}$.

В качестве основного варианта на первом этапе исследований мы полагаем (условие 2), что свободные бесконечнокомпонентные бозонные поля описываются дифференциальными уравнениями первой степени. Вполне возможно, что на каком-то этапе от этого описания нужно будет отказаться в пользу уравнений второй степени, но, зная структуру уравнений первой степени, мы без труда получим и некоторый класс уравнений второй степени.

Очевидно, что лагранжиан слабого взаимодействия с участием рассматриваемых бесконечнокомпонентных полей не может быть инвариантным относительно преобразований вторичной симметрии, порождаемой полярным или аксиальным 4-векторным представлением группы $L^{\uparrow}$. Однако и этот, и полный лагранжианы будут обладать вполне определенной вторичной симметрией, порождаемой тензором второго ранга $(0,3)$ или $(-1,2) \oplus(1,2)$ группы $L_{+}^{\uparrow}$. Можно было бы с самого начала находить все варианты теории, обладающей именно этой вторичной симметрией, но это была бы концептуально несколько иная и, возможно, более сложная задача.

Поскольку для элементов $g$ собственной группы Лоренца соотношения (5) для операторов $D^{\mu}$ и (13) для операторов $\Gamma^{\mu}$ одинаковы, то матричные операторы $D^{\mu}$ описываются формулами, аналогичными (15) и (18)-(23), произвольные величины в которых будем обозначать через $d_{\tau^{\prime} \tau} \equiv d\left(l_{0}^{\prime}, l_{1}^{\prime} ; l_{0}, l_{1}\right)$. Что касается свойств оператора $D^{0}$ относительно пространственного отражения, то они описываются соотношением, аналогичным (16), если параметр $\theta_{\mu}$ в преобразовании (2) - полярный 4-вектор, или формулой $\left\{P, D^{0}\right\}=0$, если $\theta_{\mu}-$ аксиальный 4-вектор.

Лагранжиан (12) будет инвариантен относительно преобразований вторичной сим- 
метрии (2), если выполнены следующие условия:

$$
\begin{aligned}
{\left[\Gamma^{\mu}, D^{\nu}\right] } & =0, \\
\left(D^{0} \Psi_{1}, \Psi_{2}\right) & =\left(\Psi_{1}, D^{0} \Psi_{2}\right) .
\end{aligned}
$$

Если в лагранжиане (12) константа $\kappa$ равна нулю, то его инвариантность относительно преобразований вторичной симметрии (2) будет обеспечена либо при выполнении условий $(26),(27)$, либо при выполнении условий

$$
\begin{aligned}
\left\{\Gamma^{\mu}, D^{\nu}\right\} & =0, \\
\left(D^{0} \Psi_{1}, \Psi_{2}\right) & =-\left(\Psi_{1}, D^{0} \Psi_{2}\right) .
\end{aligned}
$$

Введем матричный оператор $\Gamma^{5}$, задаваемый формулой

$$
\Gamma^{5} \xi_{\left(l_{0}, l_{1}\right) l m}=(-1)^{l_{0}+l_{1}} \xi_{\left(l_{0}, l_{1}\right) l m} .
$$

Легко убедиться, что любой 4-векторный оператор $V^{\mu}$ (подчиняющийся условию типа (5)) антикоммутирует с оператором $\Gamma^{5}$. Следовательно, если операторы $V^{\mu}$ и $W^{\nu}$ коммутируют друг с другом, то операторы $V^{\mu}$ и $\Gamma^{5} W^{\nu}$ антикоммутируют, т.е. решив уравнения (26), мы будем знать и решения уравнений (28) и наоборот. Поэтому в дальнейшем речь будет идти только об уравнениях (26).

Из условий (26) выделим два независимых соотношения:

$$
\begin{aligned}
& {\left[\Gamma^{0}, D^{0}\right]=0} \\
& {\left[\Gamma^{0}, D^{3}\right]=0}
\end{aligned}
$$

Остальные уравнения (26) являются следствием формул $(30),(31)$ и коммутационных соотношений типа (15) и (6).

Учтем, что $D^{3}=\left[I^{30}, D^{0}\right]$, и воспользумся формулами $(7),(8)$ и $(18)-(23)$. Тогда соотношения (30), (31) сведутся к алгебраической системе уравнений относительно величин $c_{\tau^{\prime} \tau}$ и $d_{\tau^{\prime} \tau}$. Заметим, что приводимые ниже уравнения $(32)-(41)$ справедливы для любого вполне приводимого представления $S$ собственной группы Лоренца, которое согласуется с условием 2 и лишь частично с условием 1 - никакие два неприводимых представления группы $L_{+}^{\uparrow}$, принадлежаших $S$, не являются эквивалентными; а "краевые" уравнения (42)-(44) имеют место для тех представлений $S$, которые к тому же удовлетворяют еше одной части условия 1 - неприводимые представления, принадлежашие $S$, конечномерны.

Для случая, когда представление $\left(l_{0}, l_{1}\right)$ содержит не менее двух значений спина $\left(\left|l_{1}\right| \geqslant\left|l_{0}\right|+2\right)$, имеем

$$
\begin{aligned}
& c\left(l_{0}+1, l_{1} ; l_{0}, l_{1}\right) d\left(l_{0}, l_{1} ; l_{0}-1, l_{1}\right)-d\left(l_{0}+1, l_{1} ; l_{0}, l_{1}\right) c\left(l_{0}, l_{1} ; l_{0}-1, l_{1}\right)=0, \\
& c\left(l_{0}, l_{1}+1 ; l_{0}, l_{1}\right) d\left(l_{0}, l_{1} ; l_{0}, l_{1}-1\right)-d\left(l_{0}, l_{1}+1 ; l_{0}, l_{1}\right) c\left(l_{0}, l_{1} ; l_{0}, l_{1}-1\right)=0, \\
& l_{1} c\left(l_{0}+1, l_{1}+1 ; l_{0}+1, l_{1}\right) d\left(l_{0}+1, l_{1} ; l_{0}, l_{1}\right)- \\
& \quad-\left(l_{0}+1\right) d\left(l_{0}+1, l_{1}+1 ; l_{0}+1, l_{1}\right) c\left(l_{0}+1, l_{1} ; l_{0}, l_{1}\right)+ \\
& \quad+l_{0} c\left(l_{0}+1, l_{1}+1 ; l_{0}, l_{1}+1\right) d\left(l_{0}, l_{1}+1 ; l_{0}, l_{1}\right)- \\
& \quad-\left(l_{1}+1\right) d\left(l_{0}+1, l_{1}+1 ; l_{0}, l_{1}+1\right) c\left(l_{0}, l_{1}+1 ; l_{0}, l_{1}\right)=0,
\end{aligned}
$$




$$
\begin{aligned}
& c\left(l_{0}+1, l_{1}+1 ; l_{0}+1, l_{1}\right) d\left(l_{0}+1, l_{1} ; l_{0}, l_{1}\right)-d\left(l_{0}+1, l_{1}+1 ; l_{0}+1, l_{1}\right) c\left(l_{0}+1, l_{1} ; l_{0}, l_{1}\right)+ \\
& \quad+c\left(l_{0}+1, l_{1}+1 ; l_{0}, l_{1}+1\right) d\left(l_{0}, l_{1}+1 ; l_{0}, l_{1}\right)- \\
& \quad-d\left(l_{0}+1, l_{1}+1 ; l_{0}, l_{1}+1\right) c\left(l_{0}, l_{1}+1 ; l_{0}, l_{1}\right)=0, \\
& l_{1} c\left(l_{0}, l_{1}+1 ; l_{0}, l_{1}\right) d\left(l_{0}, l_{1} ; l_{0}+1, l_{1}\right)+l_{0} d\left(l_{0}, l_{1}+1 ; l_{0}, l_{1}\right) c\left(l_{0}, l_{1} ; l_{0}+1, l_{1}\right)- \\
& \quad-\left(l_{0}+1\right) c\left(l_{0}, l_{1}+1 ; l_{0}+1, l_{1}+1\right) d\left(l_{0}+1, l_{1}+1 ; l_{0}+1, l_{1}\right)- \\
& \quad-\left(l_{1}+1\right) d\left(l_{0}, l_{1}+1 ; l_{0}+1, l_{1}+1\right) c\left(l_{0}+1, l_{1}+1 ; l_{0}+1, l_{1}\right)=0, \\
& c\left(l_{0}, l_{1}+1 ; l_{0}, l_{1}\right) d\left(l_{0}, l_{1} ; l_{0}+1, l_{1}\right)-d\left(l_{0}, l_{1}+1 ; l_{0}, l_{1}\right) c\left(l_{0}, l_{1} ; l_{0}+1, l_{1}\right)+ \\
& \quad+c\left(l_{0}, l_{1}+1 ; l_{0}+1, l_{1}+1\right) d\left(l_{0}+1, l_{1}+1 ; l_{0}+1, l_{1}\right)- \\
& \quad-d\left(l_{0}, l_{1}+1 ; l_{0}+1, l_{1}+1\right) c\left(l_{0}+1, l_{1}+1 ; l_{0}+1, l_{1}\right)=0, \\
& c\left(l_{0}, l_{1} ; l_{0}-1, l_{1}\right) d\left(l_{0}-1, l_{1} ; l_{0}, l_{1}\right)-d\left(l_{0}, l_{1} ; l_{0}-1, l_{1}\right) c\left(l_{0}-1, l_{1} ; l_{0}, l_{1}\right)+ \\
& \quad+c\left(l_{0}, l_{1} ; l_{0}+1, l_{1}\right) d\left(l_{0}+1, l_{1} ; l_{0}, l_{1}\right)-d\left(l_{0}, l_{1} ; l_{0}+1, l_{1}\right) c\left(l_{0}+1, l_{1} ; l_{0}, l_{1}\right)+ \\
& \quad+c\left(l_{0}, l_{1} ; l_{0}, l_{1}-1\right) d\left(l_{0}, l_{1}-1 ; l_{0}, l_{1}\right)-d\left(l_{0}, l_{1} ; l_{0}, l_{1}-1\right) c\left(l_{0}, l_{1}-1 ; l_{0}, l_{1}\right)+ \\
& \quad+c\left(l_{0}, l_{1} ; l_{0}, l_{1}+1\right) d\left(l_{0}, l_{1}+1 ; l_{0}, l_{1}\right)-d\left(l_{0}, l_{1} ; l_{0}, l_{1}+1\right) c\left(l_{0}, l_{1}+1 ; l_{0}, l_{1}\right)=0
\end{aligned}
$$

$$
\begin{aligned}
\left(l_{0}-1\right) & {\left[c\left(l_{0}, l_{1} ; l_{0}-1, l_{1}\right) d\left(l_{0}-1, l_{1} ; l_{0}, l_{1}\right)+d\left(l_{0}, l_{1} ; l_{0}-1, l_{1}\right) c\left(l_{0}-1, l_{1} ; l_{0}, l_{1}\right)\right]-} \\
& -\left(l_{0}+1\right)\left[c\left(l_{0}, l_{1} ; l_{0}+1, l_{1}\right) d\left(l_{0}+1, l_{1} ; l_{0}, l_{1}\right)+\right. \\
& \left.+d\left(l_{0}, l_{1} ; l_{0}+1, l_{1}\right) c\left(l_{0}+1, l_{1} ; l_{0}, l_{1}\right)\right]+ \\
& +\left(l_{1}-1\right)\left[c\left(l_{0}, l_{1} ; l_{0}, l_{1}-1\right) d\left(l_{0}, l_{1}-1 ; l_{0}, l_{1}\right)+\right. \\
& \left.+d\left(l_{0}, l_{1} ; l_{0}, l_{1}-1\right) c\left(l_{0}, l_{1}-1 ; l_{0}, l_{1}\right)\right]- \\
& -\left(l_{1}+1\right)\left[c\left(l_{0}, l_{1} ; l_{0}, l_{1}+1\right) d\left(l_{0}, l_{1}+1 ; l_{0}, l_{1}\right)+\right. \\
& \left.+d\left(l_{0}, l_{1} ; l_{0}, l_{1}+1\right) c\left(l_{0}, l_{1}+1 ; l_{0}, l_{1}\right)\right]=0,
\end{aligned}
$$

$l_{1}\left[c\left(l_{0}, l_{1} ; l_{0}-1, l_{1}\right) d\left(l_{0}-1, l_{1} ; l_{0}, l_{1}\right)+d\left(l_{0}, l_{1} ; l_{0}-1, l_{1}\right) c\left(l_{0}-1, l_{1} ; l_{0}, l_{1}\right)-\right.$

$\left.-c\left(l_{0}, l_{1} ; l_{0}+1, l_{1}\right) d\left(l_{0}+1, l_{1} ; l_{0}, l_{1}\right)-d\left(l_{0}, l_{1} ; l_{0}+1, l_{1}\right) c\left(l_{0}+1, l_{1} ; l_{0}, l_{1}\right)\right]+$

$+l_{0}\left[c\left(l_{0}, l_{1} ; l_{0}, l_{1}-1\right) d\left(l_{0}, l_{1}-1 ; l_{0}, l_{1}\right)+d\left(l_{0}, l_{1} ; l_{0}, l_{1}-1\right) c\left(l_{0}, l_{1}-1 ; l_{0}, l_{1}\right)-\right.$

$\left.-c\left(l_{0}, l_{1} ; l_{0}, l_{1}+1\right) d\left(l_{0}, l_{1}+1 ; l_{0}, l_{1}\right)-d\left(l_{0}, l_{1} ; l_{0}, l_{1}+1\right) c\left(l_{0}, l_{1}+1 ; l_{0}, l_{1}\right)\right]=0$,

$$
\begin{aligned}
& +l_{0}\left(l_{0}+1\right)\left[c\left(l_{0}, l_{1} ; l_{0}+1, l_{1}\right) d\left(l_{0}+1, l_{1} ; l_{0}, l_{1}\right)-\right. \\
& \left.-d\left(l_{0}, l_{1} ; l_{0}+1, l_{1}\right) c\left(l_{0}+1, l_{1} ; l_{0}, l_{1}\right)\right]+ \\
& +l_{1}\left(l_{1}-1\right)\left[c\left(l_{0}, l_{1} ; l_{0}, l_{1}-1\right) d\left(l_{0}, l_{1}-1 ; l_{0}, l_{1}\right)-\right. \\
& \left.-d\left(l_{0}, l_{1} ; l_{0}, l_{1}-1\right) c\left(l_{0}, l_{1}-1 ; l_{0}, l_{1}\right)\right]+ \\
& +l_{1}\left(l_{1}+1\right)\left[c\left(l_{0}, l_{1} ; l_{0}, l_{1}+1\right) d\left(l_{0}, l_{1}+1 ; l_{0}, l_{1}\right)-\right. \\
& \left.-d\left(l_{0}, l_{1} ; l_{0}, l_{1}+1\right) c\left(l_{0}, l_{1}+1 ; l_{0}, l_{1}\right)\right]=0,
\end{aligned}
$$$$
l_{0}\left(l_{0}-1\right)\left[c\left(l_{0}, l_{1} ; l_{0}-1, l_{1}\right) d\left(l_{0}-1, l_{1} ; l_{0}, l_{1}\right)-d\left(l_{0}, l_{1} ; l_{0}-1, l_{1}\right) c\left(l_{0}-1, l_{1} ; l_{0}, l_{1}\right)\right]+
$$

причем величины $c_{\tau^{\prime} \tau}$ и $d_{\tau^{\prime} \tau}$, которые могли бы описывать зацепление конечномерных и бесконечномерных неприводимых представлений группы $L_{+}^{\uparrow}$, должны быть опушены в 
соответствии с ранее сделанным замечанием. Кроме соотношений (32)-(41) имеется еше шесть уравнений, которые получаются из (32)-(37) заменой $c_{\tau^{\prime} \tau} \rightarrow c_{\tau \tau^{\prime}}, d_{\tau^{\prime} \tau} \rightarrow d_{\tau \tau^{\prime}}{ }^{2)}$.

Если $l_{1}=\left|l_{0}\right|+1$, то величины $c_{\tau^{\prime} \tau}$ и $d_{\tau^{\prime} \tau}$ должны удовлетворять следуюшим "краевым" уравнениям:

$$
\begin{aligned}
& c\left(l_{0} \pm 1,\left|l_{0}\right|+2 ; l_{0},\left|l_{0}\right|+2\right) d\left(l_{0},\left|l_{0}\right|+2 ; l_{0},\left|l_{0}\right|+1\right)+ \\
& \quad+d\left(l_{0} \pm 1,\left|l_{0}\right|+2 ; l_{0},\left|l_{0}\right|+2\right) c\left(l_{0},\left|l_{0}\right|+2 ; l_{0},\left|l_{0}\right|+1\right)=0, \\
& c\left(l_{0},\left|l_{0}\right|+1 ; l_{0} \mp 1,\left|l_{0}\right|+1\right) d\left(l_{0} \mp 1,\left|l_{0}\right|+1 ; l_{0},\left|l_{0}\right|+1\right)+ \\
& \quad+d\left(l_{0},\left|l_{0}\right|+1 ; l_{0} \mp 1,\left|l_{0}\right|+1\right) c\left(l_{0} \mp 1,\left|l_{0}\right|+1 ; l_{0},\left|l_{0}\right|+1\right)+ \\
& \quad+c\left(l_{0},\left|l_{0}\right|+1 ; l_{0},\left|l_{0}\right|+2\right) d\left(l_{0},\left|l_{0}\right|+2 ; l_{0},\left|l_{0}\right|+1\right)+ \\
& \quad+d\left(l_{0},\left|l_{0}\right|+1 ; l_{0},\left|l_{0}\right|+2\right) c\left(l_{0},\left|l_{0}\right|+2 ; l_{0},\left|l_{0}\right|+1\right)=0, \\
& l_{0}\left[c\left(l_{0},\left|l_{0}\right|+1 ; l_{0} \mp 1,\left|l_{0}\right|+1\right) d\left(l_{0} \mp 1,\left|l_{0}\right|+1 ; l_{0},\left|l_{0}\right|+1\right)-\right. \\
& \left.\quad-d\left(l_{0},\left|l_{0}\right|+1 ; l_{0} \mp 1,\left|l_{0}\right|+1\right) c\left(l_{0} \mp 1,\left|l_{0}\right|+1 ; l_{0},\left|l_{0}\right|+1\right)\right]- \\
& \quad-\left(l_{0}+1\right)\left[c\left(l_{0},\left|l_{0}\right|+1 ; l_{0},\left|l_{0}\right|+2\right) d\left(l_{0},\left|l_{0}\right|+2 ; l_{0},\left|l_{0}\right|+1\right)-\right. \\
& \left.-d\left(l_{0},\left|l_{0}\right|+1 ; l_{0},\left|l_{0}\right|+2\right) c\left(l_{0},\left|l_{0}\right|+2 ; l_{0},\left|l_{0}\right|+1\right)\right]=0,
\end{aligned}
$$

где верхний знак отвечает случаю $l_{0} \geqslant 0$, а нижний - случаю $l_{0} \leqslant 0$. K ним нужно прибавить еше одно уравнение, получаюшееся из (42) заменой $c_{\tau^{\prime} \tau} \rightarrow c_{\tau \tau^{\prime}}, d_{\tau^{\prime} \tau} \rightarrow d_{\tau \tau^{\prime}}$.

Нетрудно проверить, что величины $c_{\tau^{\prime} \tau}$ и $d_{\tau^{\prime} \tau}$, которые в дальнейшем будут вьписаны в следствиях 1-4, удовлетворяют всем уравнениям (32)-(44), а также обеспечивают необходимые трансформационные свойства операторов $\Gamma^{\mu}$ и $D^{\mu}$ относительно пространственного отражения и выполнение соотношений (14) и (27). Доказательство же того, что следствия 1-4 исчерпывают все варианты теории, удовлетворяющей условиям 1-4, слишком громоздко, поэтому мы ограничимся лишш его канвой, изложив в конце этого раздела некоторые общие выводы, а в начале следующего - конкретные, что позволит воспроизвести доказательство в полном объеме.

Простой анализ системы (32)-(37) и (42) показывает, что равенство нулю всех величин $c_{\tau \tau^{\prime}} d_{\tau^{\prime} \tau}$ возможно только тогда, когда или лагранжиан поля (12) расщепляем, или преобразование вторичной симметрии тривиально, что противоречит или условию 2 , или условию 3.

Из-за эквивалентности неприводимых представлений $\left(l_{0}, l_{1}\right)$ и $\left(-l_{0},-l_{1}\right)$ мы вправе ограничиться лишш такими парами чисел $\left(l_{0}, l_{1}\right)$, что $l_{1}>0$.

Пусть для некоторого представления $S$ собственной группы Лоренца, удовлетворяюшего условию 1 , сушествует решение системы (32)-(44), согласуюшееся с условиями 2, 3. Тогда среди представлений $\left(l_{0}, l_{1}\right) \in S$ имеется такое $\left(k_{0}, k_{1}\right)$, что или $c\left(k_{0}, k_{1}+1 ; k_{0}, k_{1}\right) d\left(k_{0}, k_{1} ; k_{0}, k_{1}+1\right) \neq 0$, или $d\left(k_{0}, k_{1}+1 ; k_{0}, k_{1}\right) c\left(k_{0}, k_{1} ; k_{0}, k_{1}+1\right) \neq 0$, а величины $c\left(l_{0}, l_{1}+1 ; l_{0}, l_{1}\right) d\left(l_{0}, l_{1} ; l_{0}, l_{1}+1\right)$ и $d\left(l_{0}, l_{1}+1 ; l_{0}, l_{1}\right) c\left(l_{0}, l_{1} ; l_{0}, l_{1}+1\right)$ равны

\footnotetext{
${ }^{2)}$ При ссылках на них мы будем давать им те же номера, но со штрихами.
} 
нулю, если $\left|l_{0}\right| \leqslant l_{1}-1, l_{1} \leqslant k_{1}-1$. Если $k_{0} \neq 0$, то из уравнений $(38)-(41)$ и $(43),(44)$ получаем

$$
\begin{aligned}
d\left(l_{0}+1, k_{1} ; l_{0}, k_{1}\right) c\left(l_{0}, k_{1} ; l_{0}+1, k_{1}\right) & =\frac{\left(k_{1}+k_{0}\right) a-\left(k_{1}-k_{0}\right) b}{2 k_{1}\left(k_{1}-l_{0}\right)\left(k_{1}+l_{0}+1\right)} \\
c\left(l_{0}+1, k_{1} ; l_{0}, k_{1}\right) d\left(l_{0}, k_{1} ; l_{0}+1, k_{1}\right) & =\frac{-\left(k_{1}-k_{0}\right) a+\left(k_{1}+k_{0}\right) b}{2 k_{1}\left(k_{1}-l_{0}\right)\left(k_{1}+l_{0}+1\right)} \\
d\left(l_{0}, k_{1}+1 ; l_{0}, k_{1}\right) c\left(l_{0}, k_{1} ; l_{0}, k_{1}+1\right) & =\frac{-\left(l_{0}+k_{0}\right) a+\left(l_{0}-k_{0}\right) b}{\left(k_{1}-l_{0}\right)\left(k_{1}-l_{0}+1\right)\left(k_{1}+l_{0}\right)\left(k_{1}+l_{0}+1\right)}, \\
c\left(l_{0}, k_{1}+1 ; l_{0}, k_{1}\right) d\left(l_{0}, k_{1} ; l_{0}, k_{1}+1\right) & =\frac{\left(l_{0}-k_{0}\right) a-\left(l_{0}+k_{0}\right) b}{\left(k_{1}-l_{0}\right)\left(k_{1}-l_{0}+1\right)\left(k_{1}+l_{0}\right)\left(k_{1}+l_{0}+1\right)}
\end{aligned}
$$

где по крайней мере одна из констант $a$ или $b$ не равна нулю, причем в $(45),(46)-k_{1}+$ $1 \leqslant l_{0} \leqslant k_{1}-2$, а в (47), (48) $\left|l_{0}\right| \leqslant k_{1}-1$. Если же $k_{0}=0$, то числители в правых частях равенств (45)-(48) нужно заменить соответственно на $k_{1}(A-B), k_{1}(A+B)$, $-k_{1} A+l_{0} B$ и $-k_{1} A-l_{0} B$, где по крайней мере одна из констант $A$ или $B$ не равна нулю.

Чтобы продвигаться дальше в анализе системы уравнений (32)-(44), введем величины $g_{\tau^{\prime} \tau}$ :

$$
d_{\tau^{\prime} \tau}=g_{\tau^{\prime} \tau} c_{\tau^{\prime} \tau}
$$

если $c_{\tau^{\prime} \tau} \neq 0$. Убедившись, что последнее неравенство выполнено, мы можем пользоваться следующими уравнениями, получаемыми соответственно из уравнений (42), $(32),(33),(34)$ и $(35),(36)$ и $(37),(34)$ и $\left(34^{\prime}\right),(36)$ и $\left(36^{\prime}\right)^{3)}$ :

$$
\begin{aligned}
g\left(l_{0}, l_{0}+2 ; l_{0}, l_{0}+1\right) & =-g\left(l_{0}+1, l_{0}+2 ; l_{0}, l_{0}+2\right), \\
g\left(l_{0}, l_{1} ; l_{0}-1, l_{1}\right) & =g\left(l_{0}+1, l_{1} ; l_{0}, l_{1}\right), \\
g\left(l_{0}, l_{1} ; l_{0}, l_{1}-1\right) & =g\left(l_{0}, l_{1}+1 ; l_{0}, l_{1}\right),
\end{aligned}
$$

$$
\left[g\left(l_{0}+1, l_{1}+1 ; l_{0}, l_{1}+1\right)-g\left(l_{0}, l_{1}+1 ; l_{0}, l_{1}\right)\right] \times
$$$$
\times\left[l_{1} g\left(l_{0}+1, l_{1} ; l_{0}, l_{1}\right)-\left(l_{0}+1\right) g\left(l_{0}+1, l_{1}+1 ; l_{0}+1, l_{1}\right)\right]=
$$$$
=\left[g\left(l_{0}+1, l_{1} ; l_{0}, l_{1}\right)-g\left(l_{0}+1, l_{1}+1 ; l_{0}+1, l_{1}\right)\right] \times
$$

$$
\begin{aligned}
& \times\left[\left(l_{1}+1\right) g\left(l_{0}+1, l_{1}+1 ; l_{0}, l_{1}+1\right)-l_{0} g\left(l_{0}, l_{1}+1 ; l_{0}, l_{1}\right)\right], \\
& {\left[g\left(l_{0}, l_{1}+1 ; l_{0}+1, l_{1}+1\right)-g\left(l_{0}+1, l_{1}+1 ; l_{0}+1, l_{1}\right)\right] \times}
\end{aligned}
$$$$
\times\left[l_{1} g\left(l_{0}, l_{1} ; l_{0}+1, l_{1}\right)+l_{0} g\left(l_{0}, l_{1}+1 ; l_{0}, l_{1}\right)\right]=
$$$$
=\left[g\left(l_{0}, l_{1} ; l_{0}+1, l_{1}\right)-g\left(l_{0}, l_{1}+1 ; l_{0}, l_{1}\right)\right] \times
$$$$
\times\left[\left(l_{1}+1\right) g\left(l_{0}, l_{1}+1 ; l_{0}+1, l_{1}+1\right)+\left(l_{0}+1\right) g\left(l_{0}+1, l_{1}+1 ; l_{0}+1, l_{1}\right)\right],
$$

$g\left(l_{0}+1, l_{1}+1 ; l_{0}, l_{1}+1\right) g\left(l_{0}, l_{1}+1 ; l_{0}, l_{1}\right) \times$

$$
\begin{aligned}
& \times\left[l_{1} g\left(l_{0}+1, l_{1} ; l_{0}, l_{1}\right)-\left(l_{0}+1\right) g\left(l_{0}+1, l_{1}+1 ; l_{0}+1, l_{1}\right)\right] \times \\
& \times\left[l_{1} g\left(l_{0}, l_{1} ; l_{0}+1, l_{1}\right)-\left(l_{0}+1\right) g\left(l_{0}+1, l_{1} ; l_{0}+1, l_{1}+1\right)\right] c\left(l_{0}, l_{1} ; l_{0}+1, l_{1}\right) \times
\end{aligned}
$$

\footnotetext{
${ }^{3)}$ См. сноску 2.
} 


$$
\begin{aligned}
& \times d\left(l_{0}+1, l_{1} ; l_{0}, l_{1}\right) c\left(l_{0}+1, l_{1} ; l_{0}+1, l_{1}+1\right) d\left(l_{0}+1, l_{1}+1 ; l_{0}+1, l_{1}\right)= \\
= & g\left(l_{0}+1, l_{1}+1 ; l_{0}+1, l_{1}\right) g\left(l_{0}+1, l_{1} ; l_{0}, l_{1}\right) \times \\
& \times\left[\left(l_{1}+1\right) g\left(l_{0}+1, l_{1}+1 ; l_{0}, l_{1}+1\right)-l_{0} g\left(l_{0}, l_{1}+1 ; l_{0}, l_{1}\right)\right] \times \\
& \times\left[\left(l_{1}+1\right) g\left(l_{0}, l_{1}+1 ; l_{0}+1, l_{1}+1\right)-l_{0} g\left(l_{0}, l_{1} ; l_{0}, l_{1}+1\right)\right] c\left(l_{0}, l_{1} ; l_{0}, l_{1}+1\right) \times \\
& \times d\left(l_{0}, l_{1}+1 ; l_{0}, l_{1}\right) c\left(l_{0}, l_{1}+1 ; l_{0}+1, l_{1}+1\right) d\left(l_{0}+1, l_{1}+1 ; l_{0}, l_{1}+1\right), \\
g\left(l_{0}+\right. & \left.1, l_{1}+1 ; l_{0}+1, l_{1}\right) g\left(l_{0}+1, l_{1}+1 ; l_{0}, l_{1}+1\right) \times \\
& \times\left[l_{1} g\left(l_{0}, l_{1} ; l_{0}+1, l_{1}\right)+l_{0} g\left(l_{0}, l_{1}+1 ; l_{0}, l_{1}\right)\right] \times \\
& \times\left[l_{1} g\left(l_{0}+1, l_{1} ; l_{0}, l_{1}\right)+l_{0} g\left(l_{0}, l_{1} ; l_{0}, l_{1}+1\right)\right] \times \\
& \times c\left(l_{0}, l_{1} ; l_{0}+1, l_{1}\right) d\left(l_{0}+1, l_{1} ; l_{0}, l_{1}\right) c\left(l_{0}, l_{1} ; l_{0}, l_{1}+1\right) d\left(l_{0}, l_{1}+1 ; l_{0}, l_{1}\right)= \\
= & g\left(l_{0}+1, l_{1} ; l_{0}, l_{1}\right) g\left(l_{0}, l_{1}+1 ; l_{0}, l_{1}\right) \times \\
& \times\left[\left(l_{1}+1\right) g\left(l_{0}, l_{1}+1 ; l_{0}+1, l_{1}+1\right)+\left(l_{0}+1\right) g\left(l_{0}+1, l_{1}+1 ; l_{0}+1, l_{1}\right)\right] \times \\
& \times\left[\left(l_{1}+1\right) g\left(l_{0}+1, l_{1}+1 ; l_{0}, l_{1}+1\right)+\left(l_{0}+1\right) g\left(l_{0}+1, l_{1} ; l_{0}+1, l_{1}+1\right)\right] \times \\
& \times c\left(l_{0}+1, l_{1} ; l_{0}+1, l_{1}+1\right) d\left(l_{0}+1, l_{1}+1 ; l_{0}+1, l_{1}\right) \times \\
& \times c\left(l_{0}, l_{1}+1 ; l_{0}+1, l_{1}+1\right) d\left(l_{0}+1, l_{1}+1 ; l_{0}, l_{1}+1\right)
\end{aligned}
$$

а также уравнениями, получаемыми из (50)-(54) заменой $g_{\tau^{\prime} \tau} \rightarrow g_{\tau \tau^{\prime}}$.

\section{4. СЛЕДСТВИЯ ДВОЙНОЙ СИММЕТРИИ ЛАГРАНЖИАНА СВОБОДНОГО ФЕРМИОННОГО ПОЛЯ}

Каковы бы ни были величины $p_{\dot{\tau} \tau}$ и $a_{\dot{\tau} \tau}$ в некотором каноническом базисе, можно указать такой новый канонический базис, в котором остаются неизменными формулы (8)-(10) и справедливы равенства

$$
\begin{aligned}
p_{\dot{\tau} \tau} & =p_{\tau \dot{\tau}}=1, \\
a_{\dot{\tau} \tau} & =a_{\tau \dot{\tau}}= \pm 1,
\end{aligned}
$$

причем для разных представлений $\tau$ знаки в правой части (58) могут быть как одинаковыми, так и разными.

Из соотношений $(24),(25)$ и условия 4 с учетом формул (20)-(23) получаем, что

$$
\begin{aligned}
& c\left(l_{0}+1, l_{1} ; l_{0}, l_{1}\right)=c\left(-l_{0}-1, l_{1} ;-l_{0}, l_{1}\right), \\
& c\left(l_{0}, l_{1} \pm 1 ; l_{0}, l_{1}\right)=c\left(-l_{0}, l_{1} \pm 1 ;-l_{0}, l_{1}\right), \\
& c\left(l_{0}+1, l_{1} ; l_{0}, l_{1}\right)=c^{*}\left(l_{0}, l_{1} ; l_{0}+1, l_{1}\right), \\
& c\left(l_{0}, l_{1}+1 ; l_{0}, l_{1}\right)=-c^{*}\left(l_{0}, l_{1} ; l_{0}, l_{1}+1\right),
\end{aligned}
$$

а также что величины $a_{\dot{\tau} \tau}$ для всех представлений $\tau$ одинаковы и без ограничения обшности их можно положить равными единище. Заметим, что последнее утверждение и соотношения (61), (62) потеряют силу, если опустить условие 4.

Рассмотрим случай, когда в преобразованиях (2) параметр $\theta_{\mu}$ - полярный 4 -вектор. 
Тогда величины $d_{\tau^{\prime} \tau}$ должны подчиняться условиям типа $(59),(60)$. Из формул (59) для $c_{\tau^{\prime} \tau}$ и $d_{\tau^{\prime} \tau}$ и из $(45),(46)$ при $l_{0}=-1 / 2$ получаем, что $a=b$, после чего из $(45)-(48)$ и (60) имеем для всех допустимых значений $l_{0}$

$$
\begin{aligned}
& g\left(l_{0}+1, k_{1} ; l_{0}, k_{1}\right)=g\left(l_{0}, k_{1} ; l_{0}+1, k_{1}\right), \\
& g\left(l_{0}, k_{1}+1 ; l_{0}, k_{1}\right)=g\left(l_{0}, k_{1} ; l_{0}, k_{1}+1\right), \\
& g\left(l_{0}, k_{1}+1 ; l_{0}, k_{1}\right)=g\left(-l_{0}, k_{1}+1 ;-l_{0}, k_{1}\right) .
\end{aligned}
$$

Мы должны по отдельности проанализировать две возможные ситуации:

$$
c\left(k_{1}, k_{1}+1 ; k_{1}-1, k_{1}+1\right) d\left(k_{1}-1, k_{1}+1 ; k_{1}, k_{1}+1\right) \neq 0
$$

или

$$
c\left(k_{1}, k_{1}+1 ; k_{1}-1, k_{1}+1\right) d\left(k_{1}-1, k_{1}+1 ; k_{1}, k_{1}+1\right)=0 .
$$

Остановимся на первой из них. Из уравнений (42) и (32) следует, что мы вправе ввести величины $g\left(l_{0} \pm 1, k_{1}+1 ; l_{0}, k_{1}+1\right)$ для всех допустимых значений $l_{0}$. Воспользовавшись вначале равенством (50) при $l_{0}=k_{1}-1$ и равенством (51) при $l_{1}=k_{1}+1$ и всех допустимых значениях $l_{0}$, находим, что система уравнений (53), (54) при $l_{0}=k_{1}-2$, $l_{1}=k_{1}$ дает два решения, устанавливаем, какова должна быть зависимость от $l_{0}$ величин $g\left(l_{0}, k_{1}+1 ; l_{0}, k_{1}\right)$ для каждого из решений, и проверяем эту зависимость методом математической индукции. Получаем, что одно из решений системы уравнений (53), (54) имеет вид

$$
g\left(l_{0}, k_{1}+1 ; l_{0}, k_{1}\right)=(-1)^{l_{0}+k_{1}-1} g_{0},
$$

где $g_{0}$ - некоторая константа. Оно находится в противоречии с соотношением (65) и должно быть отброшено. Тогда остается только решение

$$
\begin{aligned}
g\left(l_{0} \pm 1, k_{1} ; l_{0}, k_{1}\right) & =g\left(l_{0} \pm 1, k_{1}+1 ; l_{0}, k_{1}+1\right)=-g\left(l_{0}, k_{1}+1 ; l_{0}, k_{1}\right)= \\
& =-g\left(l_{0}, k_{1} ; l_{0}, k_{1}+1\right)=g_{0} .
\end{aligned}
$$

Используя соотношения (45)-(48) (при $a=b$ ) и (69), обрашаемся к системе уравнений $(55),(56)$ при $l_{0}=k_{1}-2, l_{1}=k_{1}$. Так как $a \neq 0$ и $g_{0} \neq 0$, то эта система непротиворечива тогда и только тогда, когда $k_{1}=3 / 2$. При таком значении $k_{1}$ имеем

$$
c\left(\frac{1}{2}, \frac{5}{2} ;-\frac{1}{2}, \frac{5}{2}\right) d\left(-\frac{1}{2}, \frac{5}{2} ; \frac{1}{2}, \frac{5}{2}\right)=d\left(\frac{1}{2}, \frac{5}{2} ;-\frac{1}{2}, \frac{5}{2}\right) c\left(-\frac{1}{2}, \frac{5}{2} ; \frac{1}{2}, \frac{5}{2}\right)=\frac{a k_{0}}{6} .
$$

Далее мы можем вновь воспользоваться системой уравнений (38)-(41) и (43), (44) при $l_{1}=k_{1}+1=5 / 2$ и т.д. Наконец, используя метод математической индукции, можно убедиться в том, что представление $S$ включает в себя все конечномерные представления группы $L_{+}^{\uparrow}$ и для всех допустимых значений $l_{0}$ и $l_{1}$ справедливы равенства

$$
\begin{aligned}
g\left(l_{0} \pm 1, l_{1} ; l_{0}, l_{1}\right) & =-g\left(l_{0}, l_{1} \pm 1 ; l_{0}, l_{1}\right)=g_{0}, \\
c\left(l_{0}, l_{1} ; l_{0}+1, l_{1}\right) c\left(l_{0}+1, l_{1} ; l_{0}, l_{1}\right) & =c\left(l_{0}, l_{1} ; l_{0}, l_{1}+1\right) c\left(l_{0}, l_{1}+1 ; l_{0}, l_{1}\right)=f_{0},
\end{aligned}
$$


где $f_{0}$ - некоторая константа. Очевидно, что решение (71) находится в противоречии с соотношениями (61), (62), связанными с условием 4, т.е. в рассматриваемой ситуации условия 1-4 не выполнимы. Если бы мы отказались от условия 4, то с учетом формул (58), (25), (20)-(23) и (71) мы нашли бы, что условия 1-3 выполняются тогда и только тогда, когда

$$
\begin{gathered}
a_{\dot{\tau} \tau}=(-1)^{l_{1}-\frac{3}{2}} e_{0}, \quad \text { если } \tau=\left(l_{0}, l_{1}\right), \\
c\left(l_{0}+1, l_{1} ; l_{0}, l_{1}\right)=c\left(l_{0}, l_{1} ; l_{0}+1, l_{1}\right)=c\left(l_{0}, l_{1}+1 ; l_{0}, l_{1}\right)=c\left(l_{0}, l_{1} ; l_{0}, l_{1}+1\right)=c_{0},
\end{gathered}
$$

где константа $e_{0}$ равна либо 1 , либо -1 , а константа $c_{0}$ вешественна. Здесь и везде в дальнейшем значения величин $c_{\tau^{\prime} \tau}$ даются с точностью до несушественных фазовых множителей, которые можно ввести или устранить, изменив относительные фазы базисных векторов пространства представления $S$.

На анализе ситуации, которая описывается равенством (67), как и на анализе всех других вариантов, мы останавливаться больше не будем.

Чтобы не загромождать формулировки следствий 1-4, отметим предварительно, что каждому представлению $S$ группы $L_{+}^{\uparrow}$, фигурируюшему в этих следствиях, соответствует однозначная (с точностью до обшего нормировочного множителя) совокупность величин $c_{\tau^{\prime} \tau}$ и $d_{\tau^{\prime}}$, выписываемая для всех подходяших пар $\tau$ и $\tau^{\prime}$, принадлежаших $S$, и однозначная (с точностью до общего знака, фиксируемого как положительный) совокупность величин $a_{\dot{\tau} \tau}: a_{\dot{\tau} \tau}=1$ для всех $\tau \in S$.

СлЕДСТВИЕ 1. Требование, чтобъ теория свободного фермионного поля удовлетворяла условиям 1-4, если параметр $\theta_{\mu}$ в преобразованиях (2) - полярный 4-вектор, выполнимо только для следующего счетного мнохества представлений $S$ собственной группь Лоренца, нумеруемых полуцелыми числами $k_{1}\left(k_{1} \geqslant\right.$ $3 / 2)$ :

$$
S^{k_{1}}=\sum_{n_{1}=0}^{+\infty} \sum_{n_{0}=-k_{1}+\frac{1}{2}}^{k_{1}-\frac{3}{2}} \oplus\left(\frac{1}{2}+n_{0}, k_{1}+n_{1}\right)
$$

причем для представления $S^{k_{1}}$ соответствующие величины $c_{\tau^{\prime} \tau} u d_{\tau^{\prime} \tau}$ даются равенствами

$$
\begin{aligned}
c\left(l_{0}+1, l_{1} ; l_{0}, l_{1}\right) & =c\left(l_{0}, l_{1} ; l_{0}+1, l_{1}\right)= \\
& =c \sqrt{\frac{\left(k_{1}-l_{0}-1\right)\left(k_{1}+l_{0}\right)}{\left(l_{1}-l_{0}\right)\left(l_{1}-l_{0}-1\right)\left(l_{1}+l_{0}\right)\left(l_{1}+l_{0}+1\right)}}, \\
c\left(l_{0}, l_{1}+1 ; l_{0}, l_{1}\right) & =c\left(l_{0}, l_{1} ; l_{0}, l_{1}+1\right)= \\
& =c \sqrt{\frac{\left(k_{1}-l_{1}-1\right)\left(k_{1}+l_{1}\right)}{\left(l_{1}-l_{0}\right)\left(l_{1}-l_{0}+1\right)\left(l_{1}+l_{0}\right)\left(l_{1}+l_{0}+1\right)}}, \\
d\left(l_{0}+1, l_{1} ; l_{0}, l_{1}\right) & =d\left(l_{0}, l_{1} ; l_{0}+1, l_{1}\right)=g_{0} c\left(l_{0}+1, l_{1} ; l_{0}, l_{1}\right), \\
d\left(l_{0}, l_{1}+1 ; l_{0}, l_{1}\right) & =d\left(l_{0}, l_{1} ; l_{0}, l_{1}+1\right)=g_{0} c\left(l_{0}, l_{1}+1 ; l_{0}, l_{1}\right),
\end{aligned}
$$

где с $с_{0}$ и $g_{0}$ - действительные константь. 
СлЕДСТвиЕ 2. Требование, чтобъ теория свободного фермионного поля удовлетворяла условиям 1-4, если параметр $\theta_{\mu}$ в преобразованиях (2) - аксиальньй 4-вектор, выполнимо только в следующих трех ситуациях:

1) для счетного мнохества представлений собственной группь Лоренца, әлемент которого дается формулой $(72)$, где $k_{1} \geqslant 3 / 2$, причем для представления $S^{k_{1}}$ величинь $c_{\tau^{\prime} \tau}$ даются формулами (73), (74) с действительной константой $c_{0}$, а величиньл $d_{\tau^{\prime} \tau}$ равньи

$$
\begin{aligned}
& d\left(l_{0}+1, l_{1} ; l_{0}, l_{1}\right)=-d\left(l_{0}, l_{1} ; l_{0}+1, l_{1}\right)=g_{0} l_{1} c\left(l_{0}+1, l_{1} ; l_{0}, l_{1}\right), \\
& d\left(l_{0}, l_{1}+1 ; l_{0}, l_{1}\right)=-d\left(l_{0}, l_{1} ; l_{0}, l_{1}+1\right)=g_{0} l_{0} c\left(l_{0}, l_{1}+1 ; l_{0}, l_{1}\right),
\end{aligned}
$$

где $g_{0}$ - действительная константа;

2) для счетного множсества представлений собственной группы Лоренца, әлемент которого дается формулой (72), где $k_{1} \geqslant 3 / 2$, причем для представления $S^{k_{1}}$ соответствующие величины $c_{\tau^{\prime} \tau} u d_{\tau^{\prime} \tau}$ даются равенствами

$$
\begin{aligned}
c\left(l_{0}+1, l_{1} ; l_{0}, l_{1}\right) & =c\left(l_{0}, l_{1} ; l_{0}+1, l_{1}\right)= \\
& =(-1)^{l_{1}-\frac{1}{2}} c_{0} l_{1} \sqrt{\frac{\left(k_{1}-l_{0}-1\right)\left(k_{1}+l_{0}\right)}{\left(l_{1}-l_{0}\right)\left(l_{1}-l_{0}-1\right)\left(l_{1}+l_{0}\right)\left(l_{1}+l_{0}+1\right)}}, \\
c\left(l_{0}, l_{1}+1 ; l_{0}, l_{1}\right) & =c\left(l_{0}, l_{1} ; l_{0}, l_{1}+1\right)= \\
& =(-1)^{l_{0}-\frac{1}{2}} c_{0} l_{0} \sqrt{\frac{\left(k_{1}-l_{1}-1\right)\left(k_{1}+l_{1}\right)}{\left(l_{1}-l_{0}\right)\left(l_{1}-l_{0}+1\right)\left(l_{1}+l_{0}\right)\left(l_{1}+l_{0}+1\right)}}, \\
d\left(l_{0}+1, l_{1} ; l_{0}, l_{1}\right) & =-d\left(l_{0}, l_{1} ; l_{0}+1, l_{1}\right)=g_{0} l_{1}^{-1} c\left(l_{0}+1, l_{1} ; l_{0}, l_{1}\right), \\
d\left(l_{0}, l_{1}+1 ; l_{0}, l_{1}\right) & =-d\left(l_{0}, l_{1} ; l_{0}, l_{1}+1\right)=g_{0} l_{0}^{-1} c\left(l_{0}, l_{1}+1 ; l_{0}, l_{1}\right),
\end{aligned}
$$

где с $c_{0}$ и $g_{0}$ - действительнье константы;

3) для представления $S$ собственной группь Лоренца, содержащего все конечномерные неприводимые представления группь $L_{+}^{\uparrow}$ с полуцельми спинами, которое будем обозначать через $S^{F}$ :

$$
S^{F}=\sum_{n_{1}=0}^{+\infty} \sum_{n_{0}=-n_{1}-1}^{n_{1}} \oplus\left(\frac{1}{2}+n_{0}, \frac{3}{2}+n_{1}\right),
$$

причем

$$
\begin{aligned}
c\left(l_{0}+1, l_{1} ; l_{0}, l_{1}\right) & =c\left(l_{0}, l_{1} ; l_{0}+1, l_{1}\right)= \\
& =(-1)^{l_{1}+\frac{1}{2}} c_{0} \sqrt{\frac{1-(-1)^{l_{1}+l_{0}}}{2\left(l_{1}-l_{0}-1\right)\left(l_{1}+l_{0}\right)}+\frac{1+(-1)^{l_{1}+l_{0}}}{2\left(l_{1}+l_{0}+1\right)\left(l_{1}-l_{0}\right)}}, \\
c\left(l_{0}, l_{1}+1 ; l_{0}, l_{1}\right) & =c\left(l_{0}, l_{1} ; l_{0}, l_{1}+1\right)= \\
& =c \sqrt{\frac{1-(-1)^{l_{1}+l_{0}}}{2\left(l_{0}-l_{1}-1\right)\left(l_{1}+l_{0}\right)}+\frac{1+(-1)^{l_{1}+l_{0}}}{2\left(l_{1}+l_{0}+1\right)\left(l_{0}-l_{1}\right)}},
\end{aligned}
$$




$$
\begin{aligned}
& d\left(l_{0}+1, l_{1} ; l_{0}, l_{1}\right)=-d\left(l_{0}, l_{1} ; l_{0}+1, l_{1}\right)=(-1)^{l_{1}+\frac{1}{2}} g_{0} c\left(l_{0}+1, l_{1} ; l_{0}, l_{1}\right), \\
& d\left(l_{0}, l_{1}+1 ; l_{0}, l_{1}\right)=-d\left(l_{0}, l_{1} ; l_{0}, l_{1}+1\right)=(-1)^{l_{0}-\frac{1}{2}} g_{0} c\left(l_{0}, l_{1}+1 ; l_{0}, l_{1}\right),
\end{aligned}
$$

где с $с_{0}$ и $g_{0}$ - действительнье константь.

\section{5. СЛЕДСТВИЯ ДВОЙНОЙ СИММЕТРИИ ЛАГРАНЖИАНА СВОБОДНОГО БОЗОННОГО ПОЛЯ}

Приводимые ниже следствия 3,4 справедливы для каждого из двух типов бозонных полей, описанных в условии 1 .

СлЕДСТвИЕ 3. Требование, чтобы теория свободного бозонного поля удовлетворяла условиям 1-4, если параметр $\theta_{\mu}$ в преобразованиях (2) - полярный 4-вектор, выполнимо только в следующих двух ситуациях:

1) для счетного множества представлений собственной группь Лоренца, нумеруемых иельми числами $k_{1}\left(k_{1} \geqslant 1\right)$ :

$$
S^{k_{1}}=\sum_{n_{1}=0}^{+\infty} \sum_{n_{0}=-k_{1}+1}^{k_{1}-1} \oplus\left(n_{0}, k_{1}+n_{1}\right),
$$

причем величиньи $c_{\tau^{\prime} \tau}$ и $d_{\tau^{\prime} \tau}$ описьваются соответственно формулами (73), (74) u (75), (76) с действительными константами с $c_{0}$ и $g_{0}$;

2) для представления собственной группь Лоренца, содержащего все конечномерные неприводимые представления группь $L_{+}^{\uparrow}$ с иельми спинами, которое будем обозначать через $S^{B}$ :

$$
S^{B}=\sum_{n_{1}=0}^{+\infty} \sum_{n_{0}=-n_{1}}^{n_{1}} \oplus\left(n_{0}, 1+n_{1}\right),
$$

причем

$$
\begin{aligned}
c\left(l_{0}+1, l_{1} ; l_{0}, l_{1}\right) & =c\left(l_{0}, l_{1} ; l_{0}+1, l_{1}\right)= \\
& =(-1)^{l_{1}+1} c_{0} \sqrt{\frac{1+(-1)^{l_{1}+l_{0}}}{2\left(l_{1}+l_{0}+1\right)\left(l_{1}-l_{0}-1\right)}+\frac{1-(-1)^{l_{1}+l_{0}}}{2\left(l_{1}+l_{0}\right)\left(l_{1}-l_{0}\right)}} \\
c\left(l_{0}, l_{1}+1 ; l_{0}, l_{1}\right) & =c\left(l_{0}, l_{1} ; l_{0}, l_{1}+1\right)= \\
& =c_{0} \sqrt{\frac{1+(-1)^{l_{1}+l_{0}}}{2\left(l_{1}+l_{0}+1\right)\left(l_{0}-l_{1}-1\right)}+\frac{1-(-1)^{l_{1}+l_{0}}}{2\left(l_{1}+l_{0}\right)\left(l_{0}-l_{1}\right)}} \\
d\left(l_{0}+1, l_{1} ; l_{0}, l_{1}\right) & =d\left(l_{0}, l_{1} ; l_{0}+1, l_{1}\right)=(-1)^{l_{1}+1} g_{0} c\left(l_{0}+1, l_{1} ; l_{0}, l_{1}\right), \\
d\left(l_{0}, l_{1}+1 ; l_{0}, l_{1}\right) & =d\left(l_{0}, l_{1} ; l_{0}, l_{1}+1\right)=(-1)^{l_{0}} g_{0} c\left(l_{0}, l_{1}+1 ; l_{0}, l_{1}\right),
\end{aligned}
$$

где с $c_{0}$ и $g_{0}-$ действительнье константы. 
СлЕДСТвиЕ 4. Требование, чтобы теория свободного бозонного поля удовлетворяла условиям 1-4, если параметр $\theta_{\mu}$ в преобразованиях (2) - аксиальный 4-вектор, выполнимо только для счетного множества представлений собственной группь Лоренца, элемент которого дается формулой (88), где $k_{1} \geqslant 2$, причем для представления $S^{k_{1}}$ величины $c_{\tau^{\prime} \tau} u d_{\tau^{\prime} \tau}$ описываются соответственно формулами (73), (74) и (77), (78) с действительными константами $c_{0}$ и $g_{0}$.

Представляется целесообразным включить в рассмотрение также варианты теории бозонного поля, когда или наряду с условием 3 , или взамен условия 3 выполняется следующее

УСловиЕ 3 А. Оба типа свободных бозонных полей, обозначаемых через $\varphi_{+}$и $\varphi_{-}$, описываются до спонтанного нарушения вторичной симметрии индентичными лагранжианами. Сумма этих лагранжианов инвариантна относительно преобразований вторичной симметрии, имеющих вид

$$
\left(\begin{array}{c}
\varphi_{+} \\
\varphi_{-}
\end{array}\right) \rightarrow\left(\begin{array}{c}
\varphi_{+}^{\prime} \\
\varphi_{-}^{\prime}
\end{array}\right)=\exp \left[-i\left(\begin{array}{cc}
0 & D^{\mu} \\
D^{\mu} & 0
\end{array}\right) \theta_{\mu}\right]\left(\begin{array}{c}
\varphi_{+} \\
\varphi_{-}
\end{array}\right)
$$

где параметры $\theta_{\mu}$ являются компонентами полярного или аксиального 4-вектора ортохронной группы Лоренца, а $D^{\mu}$ - матричные операторы.

Очевидно, что если в преобразовании (94) параметр $\theta_{\mu}$ - полярный (аксиальный) 4-вектор, то теория свободных бозонных полей удовлетворяет условиям 1, 2, 3А и 4 для тех представлений $S$ и соответствуюших им величин $c_{\tau^{\prime}} \tau$ и $d_{\tau^{\prime}}$, которые описаны в следствии 4 (следствии 3 ).

\section{6. ЗАКЛЮЧИТЕЛЬНЫЕ ЗАМЕЧАНИЯ}

В каждом из вариантов теории, описанных в следствиях $1-4$, операторы $D^{\mu}$ из преобразований вторичной симметрии (2) определены однозначно с точностью до общей нормировочной константы, а значит, группа вторичной симметрии, задаваемая преобразованиями (2) и их произведениями, тоже определена однозначно. В настоящей работе не было нужды выяснять характеристики алгебры Ли какой-либо из этих групп. Заметим лишш, что отвечаюшая следствиям 1, 2 (п. 2), 3 (п. 1) алгебра Ли группы двойной симметрии совпадает с алгеброй Ли группы Пуанкаре, однако операторы $D^{\mu}$ нельзя отождествить с генераторами сдвигов $P^{\mu}$, так как $D^{\mu}$ действуют только на спиновые переменные поля, а $P^{\mu}$ на них не действуют.

Лагранжиану (12) отвечает уравнение типа Гельфанда-Яглома (1), в котором $R=$ $\kappa E$, где $E$ - единичный оператор. Пусть $\lambda$ - собственное значение оператора $\Gamma^{0}$ :

$$
\Gamma^{0} \Psi(\lambda)=\lambda \Psi(\lambda)
$$

Тогда спектр масс $M$, получаемый из уравнения (1), связан со спектром собственных значений оператора $\Gamma^{0}$ соотношением $M=\kappa / \lambda$. Так как из-за вторичной симметрии теории оператор $\Gamma^{0}$ коммутирует с оператором $D^{3}$, который согласно равенству, аналогичному (17), и соотношениям (7), (8) переводит состояние с определенным спином $l$ 
в состояние со спинами $l-1, l$ и $l+1$, то собственными векторами оператора $\Gamma^{0}$ с собственным значением $\lambda$ будут также векторы $\left(D^{3}\right)^{n} \Psi(\lambda), n \geqslant 1$. Таким образом, спектр масс, отвечающий каждому из бесконечнокомпонентных полей изучаемой теории, будет бесконечно вырожден по спину. Детальный анализ показывает, что спектр масс к тому же непрерывен, дискретные точки в нем отсутствуют. Но уже бесконечной вырожденности по спину достаточно, чтобы поставить вопрос о спонтанном нарушении вторичной симметрии.

Благодарности. Я очень признателен А. У. Климыку, А. А. Комару и В. И. Фущичу за полезные обсуждения затронутых в настоящей работе вопросов.

\section{Список литературы}

[1] В. Л. Гинзбург, И. Е. Тамм. ЖЭТФ. 1947. Т. 17. С. 227.

[2] И. М. Гельфанд, А. М. Яглом. ЖЭТФ. 1948. Т. 18. С. 703.

[3] А. А. Комар, Л. М. Сладь. ТМФ. 1969. Т. 1. С. 50.

[4] I. T. Grodsky, R. F. Streater. Phys. Rev. Lett. 1968. V. 20. P. 695.

[5] Н. Н. Боголюбов, А. А. Логунов, А. И. Оксак, И. Т. Тодоров. Общие принципы квантовой теории поля. М.: Наука, 1987.

[6] И. М. Гельфанд, А. М. Яглом. ЖЭТФ. 1948. Т. 18. С. 1094.

[7] V. Bargmann. Math. Rev. 1949. V. 10. P. 583; P. 584

[8] E. Abers, I. T. Grodsky, R. E. Norton. Phys. Rev. 1967. V. 159. P. 1222.

[9] P. A. M. Dirac. Proc. Roy. Soc. A. 1971. V. 322. P. 435.

[10] Л. М. Сладь. ТМФ. 1970. Т. 2. С. 67.

[11] В. Паули. Принцип запрета, группа Лоренца, отражение пространства, времени и заряда. В сб.: Нильс Бор и развитие физики. Ред. В. Паули при участии Л. Розенфельда и В. Вайскопфа. М.: ИЛ, 1958. С. 46.

[12] L. M. Slad. Mod. Phys. Lett. A. 2000. V. 15. P. 379.

[13] И. М. Гельфанд, Р. А. Минлос, З. Я. Шапиро. Представления группы вращений и группы Лоренца, их применения. М.: Физматгиз, 1958. 